\title{
Tumor sólido pseudopapilar de páncreas: Tumor de Frantz
}

Fernando Barreda Bolaños ${ }^{1, a}$, Humberto Liu Bejarano ${ }^{1, a}$, Lidia Rodriguez Briceño ${ }^{1, b}$, Bruno Salmon Alva ${ }^{1, b}$, Edith Sulca Flores $^{1, b}$, Luis Taxa Rojas ${ }^{1, c}$, Francisco Berrospi Espinoza ${ }^{1, d}$

\section{RESUMEN}

El tumor pseudopapilar de páncreas, también conocido como tumor de Frantz, es una enfermedad muy rara en nuestro medio, comprende menos del $3 \%$ de los tumores pancreáticos ${ }^{(1)}$. Su etiología es desconocida, y su incidencia se observa en mujeres jóvenes con predominancia de la tercera década de la vida.

Presentamos el caso de una mujer de 23 años que ingresa por epigastralgia urente desde hace 3 meses, además de llenura precoz. Al examen presenta dolor a la palpación profunda. Cuenta con perfil bioquímico, hepático, marcadores tumorales dentro de valores normales, la tomografía espiral multicorte (TEM) abdomino-pélvica reporta lesión neoformativa sólida quística de morfología redondeada de bordes bien definidos, la patología confirma tumor sólido pseudopapilar de páncreas. La paciente se somete a resección de tumor, con evolución favorable.

Palabras clave: Tumor sólido pseudopapilar; páncreas; tumor de Frantz (Fuente: DeCS BIREME).

\section{Solid pseudopapillary tumor of the pancreas: Frantz's tumor}

\section{ABSTRACT}

The solid pseudopapillary tumor of the pancreas, also known as Frantz's tumor, is a very rare disease in our country, comprising less than $3 \%$ of pancreatic tumors ${ }^{(1)}$. Its etiology is unknown and it is predominantly found in young women in their third decade of life.

We present the case of a 23-year-old woman who was admitted due to a 3-month burning epigastralgia and early satiety. On examination, she had pain with deep palpation. Her biochemical profile, liver profile, and tumor markers were within normal values. An abdomen and pelvis multislice helical/spiral computed tomography (CT) scan showed a solid cystic neoformative lesion with rounded structure and well-defined borders. Pathology confirmed a solid pseudopapillary tumor of the pancreas. The patient underwent a tumor resection with favorable evolution.

Keywords: Solid pseudopapillary tumor; pancreas; Frantz's tumor (Source: MeSH NLM).

1. Instituto de Enfermedades Neoplásicas. Lima, Perú.

a. Médico asistente Servicio de Gastroenterologia.

b. Médico residente de Gastroenterología.

c. Médico asistente Departamento de Patología.

d. Médico asistente Departamento de Abdomen. 


\section{INTRODUCCIÓN}

El tumor sólido pseudopapilar de páncreas o tumor de Frantz, es una enfermedad muy rara en nuestro medio, fue descrito por primera vez en 1959 por el patólogo Virginia Kneeland Frantz (1). En 1996, la organización mundial de la salud lo denominó tumor sólido pseudopapilar ${ }^{(2)}$.

Comprende sólo 0.2 a $2.7 \%$ de todos los tumores del páncreas ${ }^{(1)}$. Su etiología es aún incierta, y suele presentarse en mujeres jóvenes entre $18 \mathrm{y}$ 35 años de edad, con una media de 24 años ${ }^{(1,2)}$. La manifestación clínica de esta enfermedad suele ser una masa abdominal de crecimiento lento con o sin dolor abdominal ${ }^{(2,3)}$. Es de bajo potencial maligno; sin embargo, algunos casos pueden ser localmente agresivos e infiltrativos, con metástasis en el hígado, pulmón y piel ${ }^{(4)}$. En el examen histológico, el tumor es una masa sólida con estructuras pseudopapilares y pseudoquísticas con microvasculatura rica en diversas proporciones ${ }^{(5)}$. La resección quirúrgica es el tratamiento de elección, y su pronóstico es excelente, con una tasa de supervivencia a cinco años tan alta como $94-97 \%$ (2).

\section{REPORTE DEL CASO}

Paciente mujer de 23 años, natural y procedente de

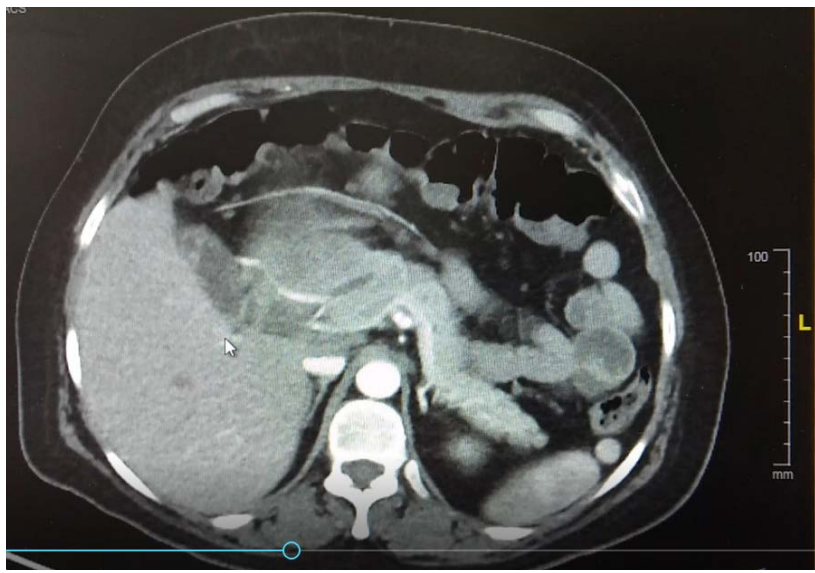

Lima, sin antecedentes de importancia. Acudió con un tiempo de enfermedad de 3 meses caracterizado por epigastralgia urente, dispepsia a grasas, llenura precoz, niega otra sintomatología. Al examen físico, funciones vitales normales, dolor a la palpación profunda en epigastrio, el resto del examen sin hallazgos significativos.

En los exámenes de laboratorio presenta hemoglobina $11.6 \mathrm{mg} / \mathrm{dl}$, normocítica normocrómica, leucocitos 8160, plaquetas 292000 , el perfil bioquímico, hepático, CA 19.9 y el antígeno carcinoembrionario dentro de los valores normales. Se realizó ecografía abdominal, observando masa con signos de necrosis central dependiente de cabeza de páncreas; TEM abdomino-pélvica mostró extensa lesión neoformativa sólida, quística, de morfología redondeada de bordes bien definidos que ejerce un efecto de masa, de aproximadamente 9 centímetros de diámetro mayor con calcificaciones periféricas y pequeños ganglios peritumorales, no se asocia a alteraciones de los planos grasos adyacentes y condiciona atrofia del cuerpo y la cola del páncreas, así como desplazamiento de las estructuras vasculares del tronco celiaco y de las estructuras adyacentes al duodeno, no se evidencia signos de infiltración (Figura 1). Cuenta con gastroscopia: compresión extrínseca a nivel de bulbo duodenal.

Figura 1. TEM abdómino pélvica: muestra extensa lesión neoformativa sólida quística de morfología redondeada de bordes bien definidos

La ecoendoscopia, informa extensa lesión sólida de aspecto heterogéneo dependiente de cabeza de páncreas, la cual se biopsia. Los resultados de histopatología de biopsia indicaron neoplasia pseudopapilar sólida de páncreas. Con inmunohistoquímica indicando beta-catenina (positivo), cromogranina (negativo), sinaptofisina (negativo) (Figura 2). 

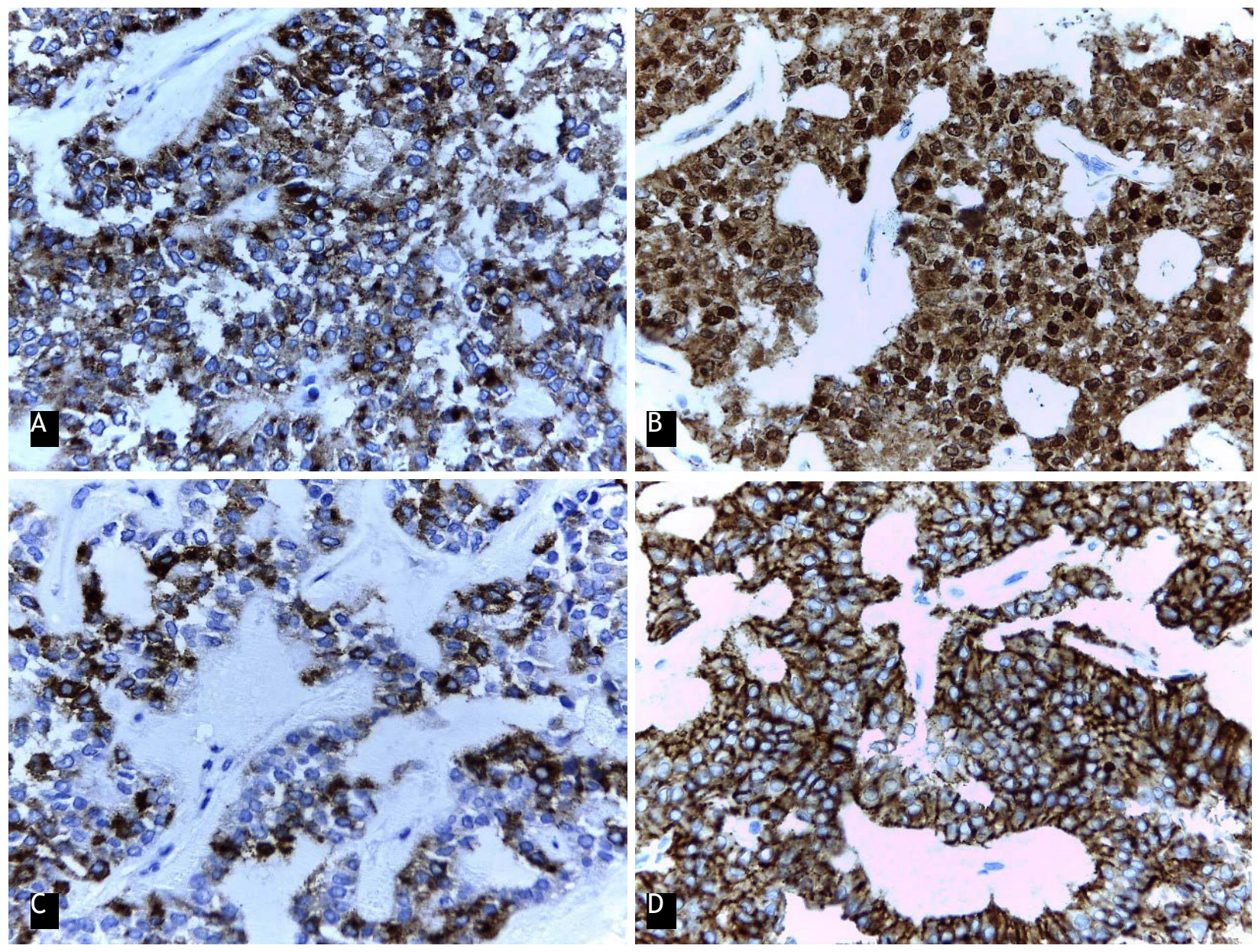

Figura 2. Inmunohistoquímica (a) La neoplasia expresa CD10, (b) Beta-catenina positivo, (c) sinaptofisina negativo, (d) CD56 positivo

Finalmente se realizó resección quirúrgica del tumor mediante laparotomía exploradora, duodenopancreatectomía proximal clásica, colecistectomía, pancreatoyeyunoanastomosis $\mathrm{T}-\mathrm{T}$ según técnica de telescopaje modificada, hepáticoyeyuno anastomosis $\mathrm{T}-\mathrm{L}$, gastroenteroanastomosis.

Dentro de los hallazgos operatorios se encontró a nivel de cabeza de páncreas tumoración sólido-quística de bordes bien definidos de $11 \times 10$ centímetros, firmemente adherida a segmento del mesocolon transverso por debajo de la arcada de Drummond. Dicha tumoración rodea menos de 180 grados la vena mesentérica superior y el tronco venoso mesentérico portal, arteria mesentérica superior desplazada por la tumoración. Cuerpo y cola de páncreas de aspecto graso blando, con Wirsung no identificable a la sección. Vesícula biliar de paredes delgadas, sin litos.

Informe anatomo-patológico de pieza operatoria grande: Tumor sólido pseudopapilar de páncreas (tumor de Frantz), no se evidencia invasión linfovascular ni perineural. Duodeno, colédoco, ganglios linfáticos y vesícula biliar libre de neoplasia maligna. Inmunohistoquímica: beta-catenina (positivo), vimentina (positivo), sinaptofisina (negativo), CD10 y CD56 positivo (Figura 3 ).

Un seguimiento de 1 año después, no mostró signos de recidiva tumoral ni insuficiencia endocrina y exocrina del páncreas. 

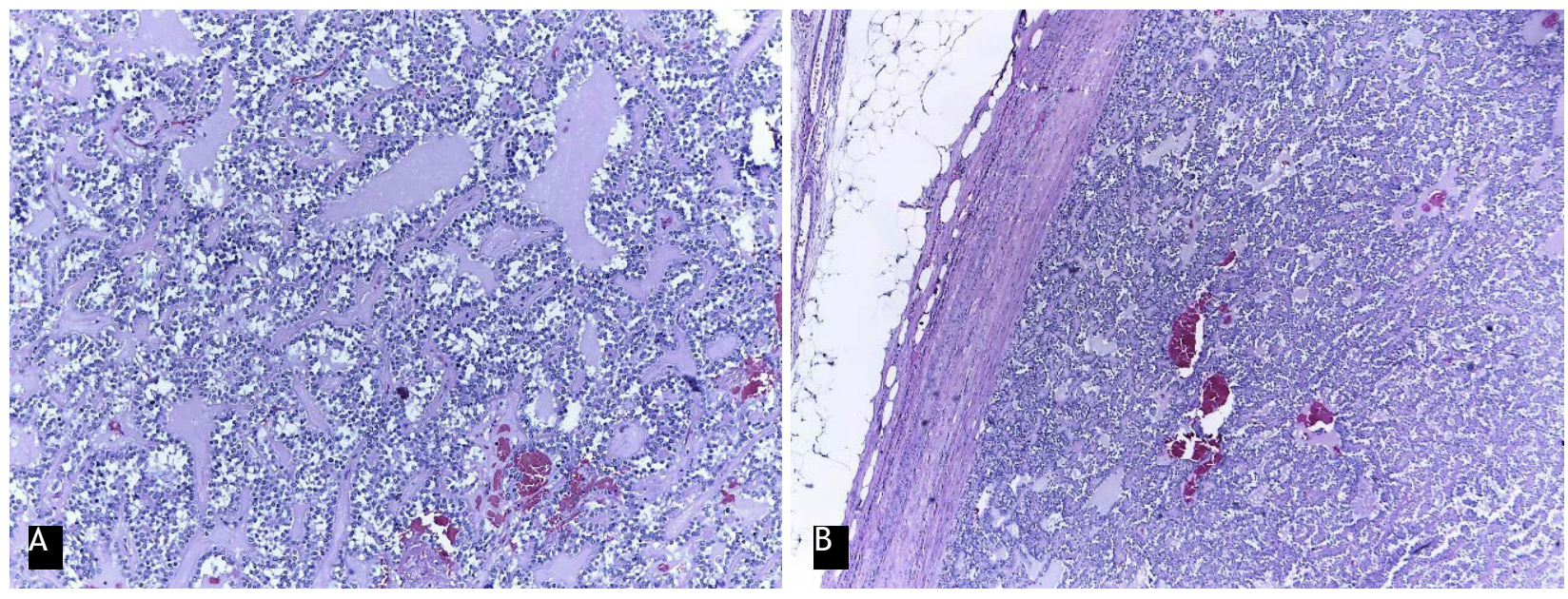

Figura 3. Informe anátomo-patológico de pieza operatoria grande (A): Se observa el patrón característico pseudopapilar; (B) neoplasia sólida pseudopapilar, a bajo poder se observa la buena delimitación de neoplasia sin extensión a los tejidos blandos peri pancreáticos

\section{DISCUSIÓN}

El tumor sólido pseudopapilar del páncreas es una patología muy rara, comprende sólo 0.2 a $2.7 \%$ de todos los tumores del páncreas; a pesar de que su etiología aún resulta desconocida se postula un origen hormonal puesto que aparecen más frecuentemente en mujeres en edad reproductiva; esta teoría se apoya además en la inmunohistoquímica (positividad para receptores de progesterona) y en la estrecha imbricación del tejido pancreático y genital durante la organogénesis precoz $(1,3,5)$.

En el 2008 un estudio reportó que en el Instituto Nacional de Enfermedades Neoplásicas (INEN) se presentaron 28 casos que representaron el $3.7 \%$ de todas las neoplasias pancreáticas, cifra discretamente superior a lo referido en la literatura mundial ( 0.13 a $2.7 \%$ ); con una relación de 6:1 a favor del género femenino, el tamaño tumoral promedio fue 10 centímetros y la localización más frecuente fue la cabeza de páncreas ${ }^{(7)}$.

La mayoría de estos tumores tienen un curso benigno, aunque en un pequeño porcentaje de los casos se ha descrito enfermedad a distancia, siendo el hígado el órgano más frecuentemente afectado, seguido de los ganglios linfáticos y el peritoneo. Se ha descrito que un $15 \%$ de pacientes presentan metástasis ${ }^{(3,4)}$. El factor clínico asociado a mal pronóstico es el sexo masculino y la edad avanzada ${ }^{(6)}$.

Generalmente, se encuentra de manera incidental en el examen radiológico por otras razones y no hay signos y síntomas clínicos típicos ${ }^{(1,5)}$. En algunos pacientes, el tumor se nota debido a dolor abdominal crónico o agudo, como en nuestro caso. Pero las características de presentación suelen ser vagas e incluyen dolor abdominal, llenura, náuseas y vómitos debido a un tumor voluminoso (tamaño medio de 11 centímetros), que comprime las estructuras locales en la parte superior del abdomen ${ }^{(5,8)}$.

Este tumor generalmente se localiza en la cabeza del páncreas. El conocimiento de las características radiológicas del tumor de Frantz es muy importante para establecer un diagnóstico preciso antes de la operación y una cirugía planificada. Las herramientas típicas utilizadas para diagnosticar son ecografía abdominal y tomografía computarizada, donde aparece como una masa sólida bien demarcada, generalmente heterogénea en la textura del eco, que a veces contiene áreas quísticas llenas de líquido hipoecoicas en ecografía. Las tomografías computarizadas muestran una masa heterogénea, a menudo con una mejora de contraste periférica correspondiente a la pseudocápsula fibrosa $\left.{ }^{(5,8,14}\right)$. En nuestro caso el tumor era sólido-quístico de morfología redondeada de bordes bien definidos que ejercía un efecto de masa.

Ante el hallazgo de una masa pancreática con componente sólido y quístico se debe hacer diagnóstico diferencial con el adenoma seroso microquístico, las neoplasias quísticas mucinosas, los tumores de islotes celulares quísticos, los pancreatoblastomas, 
los carcinomas de células acinares, los tumores mucosecretores, el hemangioma vascular «tumorlike» y los pseudoquistes hemorrágicos calcificados. Estas 3 últimas entidades son poco frecuentes en pacientes menores de 30 años ${ }^{(8)}$.

Con respecto al uso de ecoendoscopía, la literatura menciona que el diagnóstico definitivo es difícil por las siguientes razones: En primer lugar, los hallazgos de la ecoendoscopia son complejos $y$, a menudo, variables según el grado de degeneración hemorrágica; en segundo lugar, la aparición de tumores sólidos pseudopapilares en pacientes varones tiende a ser una lesión pequeña y sólida sin componente quístico, y los tumores pequeños con frecuencia tienden a presentarse como masas sólidas puras con un margen agudo; en tercer lugar, los hallazgos de la Eco endoscopia de los tumores pseudopapilares de $<3$ centímetros parecen diferir de aquellos que son $>3$ centímetros. Proponen que el patrón de eco interno que podría ayudar a diferenciar sobre todo con los tumores neuroendocrinos, es el patrón heterogéneo prominente ${ }^{(9,10)}$.

En la paciente se realizó biopsia dirigida por ecoendoscopia alta, y los resultados fueron neoplasia pseudopapilar sólida de páncreas.

La evaluación histológica muestra estructuras típicamente sólidas y pseudopapilares, vascularización intensiva o estructuras pseudoquísticas hemorrágicas en diversas proporciones. En nuestro caso se observó una neoplasia sólida pseudopapilar, que a bajo poder se observa la buena delimitación de neoplasia sin extensión a los tejidos blandos peripancreáticos; y que a alto poder se observa que las células neoplásicas tienen hendidura nuclear, pueden mostrar vacuolización citoplasmática y se pueden observar glóbulos eosinofílicos.

El inmunofenotipo de esta neoplasia expresa múltiples proteínas, pero estas no son específicas para el tumor, la beta-catenina es la más característica ${ }^{(3,6)}$. Los marcadores neuroendocrinos (CD10, CD56, enolasa neuronal específica, cromogranina A, sinaptofisina), marcador mesenquimal (vimentina), marcador epitelial (citoqueratina) y marcador relacionado con la hormona (receptor de progesterona) deben considerarse durante el diagnóstico. Las características inmunohistoquímicas respaldan la idea de que el tumor se origina en células multipotentes que no se han diferenciado en células endocrinas o exocrinas ${ }^{(3,5,13)}$.

El tratamiento, tanto de la patología primaria como de su diseminación y recurrencia, es quirúrgico ${ }^{(14)}$. La resección local es, por lo tanto, la terapia de elección. La pancreatectomía distal con o sin preservación esplénica se puede realizar para los tumores en el tronco o la cola del páncreas, y la neurodesnectomía del páncreas para los tumores de la cabeza del páncreas ${ }^{(11)}$.

En el caso presentado se realizó resección quirúrgica del tumor mediante laparotomía exploradora, duodenopancreatectomia proximal clásica, colecistectomía, pancreatoyeyunoanastomosis T-T según técnica de telescopaje modificada, hepático yeyuno anastomosis T-L, gastroentero anastomosis.

Las descripciones de los informes de casos relacionados con la resección incompleta del tumor de Frantz entre 1985 y $2008{ }^{(7)}$ muestran que a pesar del bajo potencial maligno, la mediana de supervivencia en pacientes sometidos a resección incompleta fue de solo 5.7 años. Es por eso que la resección completa del tumor de Frantz siempre está justificada, incluso al precio de una cirugía de mutilación difícil. En caso de tumores irresecables, la literatura menciona el uso de quimioterapia y radioterapia ${ }^{(12,13,14)}$.

La tasa de supervivencia a cinco años informada es tan alta como $94-97 \%^{(2,4)}$.

En nuestra paciente no se ha evidenciado recidiva, en este primer año de seguimiento.

\section{REFERENCIAS BIBLIOGRÁFICAS}

1. Serdar K, Abuzer D, Vural S, Suleyman K, Veysel E, Mustafa A. A pancreatic pseudopapillary tumor enucleated curatively. Int J Surg Case Resp. 2015; 10: 118-120.

2. Law J, Ahmed A, Singh V, Akshintala V, Olson $M$, Raman $S$, et al. A systematic review of solid pseudopapillary neoplasm: Are these rare lesions?. Pancreas. 2014; 43(3): 331-337.

3. Wang P, Wei J, Wu J, Xu W, Chen Q, Gao W, et al. Diagnosis and treatment of solid-pseudopapillary tumors of the pancreas: A single institution experience with 97 cases. Pancreatology. 2018. Disponible en: http://www.pancreatology.net/ article/S1424-3903(17)30901-8/references

4. Hao Wu, Yan Fen Huang, Xiang Hong, Mei Hua Xu. Extrapancreatic solid pseudopapillary neoplasm follower by multiple metastases: case report. World J Gastrointest Oncol. 2017; 9(12): 497-501.

5. Blanco Faramiñan, Zamora Santana. La neoplasia solida-pseudopapilar del páncreas como entidad enigmática. Rev Cubana Cir. 2015; 54(3): 285-298.

6. He Song, Ming Dong, Jianping Zhou, Weiwei Sheng, 
Banghua Zhong, Wei Gao. Solid Pseudopapillary neoplasm of the páncreas: Clinicopathologic feature, risk factors of malignancy, and survival of 53 cases from a single center. BioMed Res Int. 2017. 1-7.

7. Abad Licham, Sanchez Lihon, Celis Zapata. Tumor sólido pseudopapilar de páncreas en el Instituto Nacional de Enfermedades Neoplásicas. Rev Gastroenterol Perú. 2008; 28(4): 356-361.

8. Lubesky N, Papoulas M, Lessing Y, Gitstein G, Brazowski E, Nachmany I, et al. Solid pseudopapillary neoplasm of the páncreas: management and long term outcome. Eur J Surg Oncol. 2017; 43 (6): 10561060.

9. Akira Aso, Ihara E, Nakamura K, Sudovykh I, Ito $T$, Nakamura $M$, et al. Solid pseudopapillary of the páncreas in Young male patients: three case reports. Hindawi. Case Rep Gastrointest Med. 2017; 2017:9071678.

10. Claudio Bravo, Sonia Vilaca, Joaquim Falcao. Solid pseudopapillary neoplasm-case report of a rare pancreatic tumor. Inter J Surg Case Rep. 2017; 33: 148-150.

11. Stowik-Moczydłowska Z, Gogolewski M, Yaqoub S, Piotrowska A, Kaminski A. Solid pseudopapillary tumor of the páncreas (Frantz tumor): two case reports and a review of the literatura. J Med Case Rep. 2015; 9(268): 1-6.

12. Ovidiu Vasile, Madalina Bota, Emilia mihut, Rares Buiga. Solid pseudopapillary tumor of the páncreas: clinical-pathological features and management of 13 cases. Clujul Med. 2017; 90(2): 171-178.

13. Namita Bhutani, Pradeep Kajal, Sham Singla, Vijender Sangwan. Solid pseudopapillary tumor of

the páncreas: experience at a tertiary care centre of northern India. Inter J Surg Case Rep. 2017; 39: 225-230.

14. Tafur Anzola A, Suarez Jimenez D. Tumor de Frantz: el tumor de las mujeres jóvenes. Correlación Radiológica - Patológica de dos casos en tomografía revisión de la literatura. Revista Med. 2017; 25(1): 70-77.

Fuentes de financiamiento:

Este artículo ha sido financiado por los autores.

Conflictos de interés:

Los autores declaran no tener ningún conflicto de interés.

\section{Correspondencia:}

Fernando Barreda Bolaños

Dirección: Scipion Llona $\mathrm{N}^{\circ} 180$ Of 603 . Miraflores

Teléfono: 998094949

Correo electrónico: Fernando.barreda73@hotmail.com

\section{Recibido: 15 de abril de 2018}

Evaluado: 25 de abril de 2018

Aprobado: 06 de mayo de 2018.

( ) La revista. Publicado por Universidad de San Martín de Porres, Perú. (cc) ву Licencia de Creative Commons Artículo en acceso abierto bajo términos de Licencia Creative Commons Atribución 4.0 Internacional. (http://creativecommons.org/licenses/by/4.0/)

\section{ORCID iDs}

Fernando barreda Bolaños Humberto Liu Bejarano Lidia Rodriguez Briceño

Bruno Salmon Alva

Edith Sulca Flores https://orcid.org/0000-002-79236299 https://orcid.org/0000-0002-8417-0837 https://orcid.org/0000-0003-1975-2889 https: / / orcid.org/0000-0003-4057-0529 https://orcid.org/0000-0002-1910-0576 